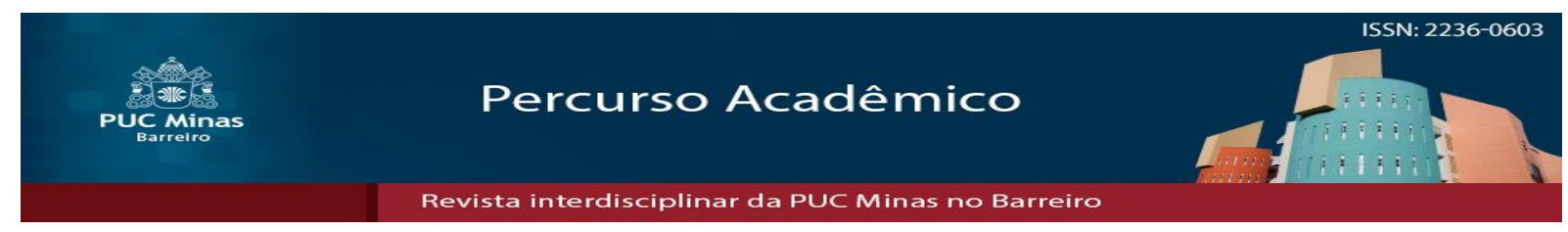

Revista interdisciplinar da PUC Minas no Barreiro

Artigo/Tema Livre

Licenca Creative Commons Attribution 4.0 International license

\title{
Tendência temporal da anemia em crianças de creches públicas de Belo Horizonte - MG
}

Temporal trend of anemia in children from public day care centers in Belo Horizonte - MG

\section{Resumo}

\author{
Maitê Costa da Silva ${ }^{1}$ \\ Flávio Diniz Capanema ${ }^{2}$ \\ Thaís de Souza Chaves de Oliveira ${ }^{3}$ \\ Daniela da Silva Rocha ${ }^{4}$ \\ Bruna Vieira de Lima Costa ${ }^{5}$ \\ Simone Cardoso Lisboa Pereira ${ }^{6}$ \\ Joel Alves Lamounier ${ }^{7}$
}

Determinar a tendência temporal da anemia em crianças de creches públicas de Belo Horizonte-MG no período de 2001 a 2011. Para tanto, foi realizada uma análise de tendência temporal da anemia determinada por série de estudo de corte transversal nos anos de 2001, 2005 e 2011, em crianças pré-escolares de seis a 72 meses pertencentes a creches públicas do Distrito Sanitário Leste de Belo Horizonte-MG. Anemia foi determinada pelo valor da hemoglobina sérica de acordo com os pontos de corte recomendados pela OMS: Hb $<11,0 \mathrm{~g} / \mathrm{dL}$ para crianças de seis a 60 meses e $\mathrm{Hb}<11,5 \mathrm{~g} / \mathrm{dL}$ para idade

Artigo recebido em 28 de Agosto de 2018 e aprovado em 04 de Junho de 2019.

${ }^{1}$ Doutora em Ciências da Saúde-UFMG, Mestre em Ciência de Alimentos- UFMG, Graduada em Nutrição pela UFVJM e atualmente Professora Adjunto do IFMG. Atualmente é Professora Adjunto do IFMG. Brasil. E-mail: maitecsilva@gmail.com

${ }^{2}$ Doutor em Medicina pela UFMG, Mestre em Ciências da Saúde pela UFMG e Graduado em Medicina pela UFMG. Brasil. E-mail: fcapa@uol.com.br

${ }^{3}$ Mestre em Ciências da Saúde pela UFMG e Graduada em Nutrição pela UFMG. Brasil. Email: orion.chaves@hotmail.com

${ }^{4}$ Doutorado e mestrado em Ciências da Saúde pela Faculdade de Medicina de Viçosa em Minas Gerais. Graduação em Nutrição pela Universidade Federal de Viçosa. Atualmente é Professora adjunta da Universidade Federal da Bahia. Brasil. E-mail: drochaufba@gmail.com

${ }^{5}$ Doutora em Enfermagem pela UFMG, Mestre em Medicina Veterinária pela UFMG, Graduada em Nutrição pela Newton Paiva. Atualmente é Professora Adjunta da UFMG. Brasil. E-mail: brunavlcosta@gmail.com

${ }^{6}$ Doutora e Mestre em Microbiologia Agrícola pela UFV e Graduada em Nutrição pela UFV. Atualmente é Professor Associado II da UFMG. Brasil. E-mail: simoneclpereira@gmail.com

${ }^{7}$ Doutorado e mestrado em Ciências da Saúde pela Faculdade de Medicina de Viçosa em Minas Gerais. Graduação em Nutrição pela Universidade Federal de Viçosa. Atualmente é Professor Titular da UFSJ. Brasil. E-mail: jalamo@medicina.ufmg.br 
superior. A tendência temporal da anemia apresentou estabilidade nos três cortes avaliados, sendo os resultados encontrados de $28,61 \%$ em 2001 , $26,78 \%$ em 2005 e 23,64\% em 2011. Não houve diferença significativa entre os percentuais de anemia por sexo. Para a faixa etária das crianças observou-se uma maior concentração de anemia na faixa etária de até 24 meses. Observouse um decréscimo da anemia com o aumento da idade. A tendência temporal da anemia apresentou valores próximos de prevalência da doença nos onze anos avaliados, demonstrando a importância da mesma como um agravo de saúde pública.

Palavras-chave: Criança. Prevalência. Anemia

\section{Abstract}

To determine temporal trend of anemia in children attended by daycare centers in the city of Belo Horizonte, Minas Gerais, in the period of 2001-2011.Temporal trend in anemia was determined by a cross-sectional study serie in the years 2001, 2005 and 2011. For the analysis it was evaluated children aged from six to 72 months enrolled in public daycare centers of Belo Horizonte, Minas Gerais. Anemia was determined by the measurement of serum hemoglobin, $\mathrm{Hb}$ $<11.0 \mathrm{~g} / \mathrm{dL}$ for children aged ranging from six to 60 months, $\mathrm{Hb}<11.5 \mathrm{~g} / \mathrm{dL}$ for children older than 60 months. Temporal trend of anemia has showed decrease of prevalence, with the results of $28.61 \%(2001), 26.78 \%(2005)$ and $23.64 \%$ (2011). There was not significant difference of anemia percentage among children genders. In relation to the age groups, it was observed higher anemia incidence for children aged until 24 months. We observed that the anemia prevalence decreased as the age was increasing. The temporal trend of anemia 
has showed similar disease prevalence within the 11 years evaluated. This study has shown the importance of anemia as a public health problem indicator.

Key-words: Children. Prevalence. Anemia

\section{1 introdução}

A anemia, condição na qual o número de hemácias ou sua capacidade de carreamento de oxigênio torna-se insuficiente para suprir as necessidades fisiológicas do organismo, mostra-se como um dos maiores problemas de Saúde Pública no mundo e segundo a Organização Mundial de Saúde (OMS) um em cada quatro indivíduos no mundo é portador de alguma forma de anemia. Ela pode ser resultante de uma ampla variedade de causas carenciais, infecciosas e inflamatórias crônicas, que podem frequentemente coexistir, sendo a deficiência de ferro sua causa mais prevalente. De acordo com o relatório "Global Burden of Disease" da Organização Mundial de Saúde (OMS) a anemia por deficiência de ferro atingiu, em 2004, um total de 1,16 bilhão de pessoas em todo o mundo (OMS, 2008; LOPEZ, et al., 2006).

Os grupos de maior vulnerabilidade para a instalação da anemia são as crianças em idade pré-escolar e gestantes, assim, a alta prevalência da anemia nestes grupos em todo o mundo, atenta para a necessidade da avaliação das medidas para o controle e combate da doença. Trabalhos recentes têm demonstrado que crianças com idade inferior a dois anos representam importante fator de risco para a ocorrência da anemia, sendo estimada prevalência superior a 60\% (MACHADO et al., 2005; SPINELLI, et al., 2005).

As conseqüências da anemia no organismo, em especial na infância, são diversas, comprometendo o desenvolvimento neuropsicomotor, o sistema imunológico, a capacidade intelectual, dentre outros. Assim, controlar a prevalência e, sobretudo evitar sua ocorrência na infância é de especial importância e justifica as inúmeras tentativas governamentais de controle da doença. No Brasil, as políticas governamentais adotadas são a obrigatoriedade de fortificação de ferro e ácido fólico nas farinhas de trigo e milho (2002) e o Programa Nacional de Suplementação de Ferro (COSTA, et al., 2009; ENSGSTROM, et al., 2009; BRASIL, 2011). 
A OMS preconiza a realização de estudos que possam demonstrar a realidade da anemia nas populações, bem como avaliar a eficácia dos programas previamente adotados. Até o presente momento poucos estudos têm sido realizados com o objetivo de avaliar a efetividade destes programas no Brasil. Dessa forma, estudos de tendência temporal poderão contribuir para que gestores públicos possam criar políticas eficazes de combate à anemia, sobretudo nas faixas etárias de maior risco (WHO, 2003; AZEREDO, et al., 2010; SILVA, et al., 2007).

Assim, a análise da tendência temporal da anemia contribui para 0 apontamento de questões socioeconômicas e demográficas que possam estar relacionadas a distúrbios nutricionais e reunir dados para o planejamento e controle dos mesmos. Nesse contexto, o presente artigo teve como objetivo avaliar a tendência temporal da anemia em crianças de creches públicas de Belo Horizonte- MG, em um período de 11 anos consecutivos.

\section{Desenvolvimento}

O presente estudo trata-se de uma análise de tendência temporal composto por três cortes transversais realizados nos anos 2001, 2005 e 2011 sobre a tendência temporal da anemia em crianças de 6 a 72 meses, matriculadas em período integral em 25 creches pertencentes ou conveniadas com a Prefeitura Municipal de Belo Horizonte - MG, localizadas na área de abrangência administrativa do Distrito Sanitário Leste. Em todas as creches foram agendadas reuniões com os pais ou responsáveis pelas crianças para esclarecimentos sobre o estudo, distribuição de folheto explicativo sobre a anemia e recolhimento da assinatura do Termo de Consentimento Livre e Esclarecido- TCLE.

O estudo seguiu as recomendações da Resolução 196/96 e foi aprovado pelo Comitê de Ética em Pesquisa da Universidade Federal de Minas GeraisUFMG (Parecer ETIC 123/00; 273/04 e 602/10), com anuência da Secretaria 
de Municipal de Educação de Belo Horizonte-MG, por meio de carta contendo consentimento para execução do projeto nas creches.

O Distrito Sanitário Leste conta com um total de aproximadamente 2.600 crianças institucionalizadas, representando $13,2 \%$ das crianças atendidas em creches públicas do município de Belo Horizonte. A população residente no Distrito Leste apresenta características heterogêneas em relação à condição socioeconômica, sendo constituída tanto por indivíduos em condições precárias de vida como por outros provenientes de famílias com bom poder aquisitivo. Porém, as crianças atendidas nas referidas creches pertencem às classes socioeconômicas de menor poder aquisitivo (SILVA; FRANCESCHINI, 2007).

A amostra foi calculada com base no número de crianças com mais de seis meses matriculadas nas já referidas creches, sendo o $N$ amostral de 2600 crianças. A prevalência da anemia foi estimada em 30\% (POLLIT, 1999), precisão de $5 \%$, intervalo de confiança de $90 \%$, além disso, foi adicionado o percentual de 20\% para compensar possíveis perdas, totalizando um $\mathrm{n}$ de 148 crianças. Dessa forma participaram do estudo em 2001 um total de 353 crianças, 407 crianças em 2005 e 165 crianças em 2011. Este cálculo foi realizado por meio do programa Statcal do software Epi-Info 6.04. As crianças foram selecionadas por randomização, com o uso do programa Excel 2003. O número de crianças sorteadas em cada creche foi estabelecido pelo peso que cada uma delas representava em relação ao total de crianças matriculadas em cada ano. Foram incluídas no estudo todas as crianças presentes no dia do exame, com ausência de sinais e sintomas de doenças infecciosas, como febre, tosse, diarréia e/ou vômitos.

Os dados de identificação (nome e data de nascimento) foram coletados das fichas de inscrição das crianças nas creches. As características de sexo e idade foram obtidas por meio de questionário padronizado aplicado em entrevistas aos pais ou responsáveis. As entrevistas foram realizadas nas creches, no horário de entrada ou saída das crianças.

Para a avaliação da anemia foi colhida amostra de sangue de cada criança por meio de punção capilar e a concentração de hemoglobina foi 
medida utilizando o fotômetro portátil hemoglobinômetro (Hemocue)®. O diagnóstico da anemia considerou os valores recomendados pela OMS (2001) sendo anêmicas crianças que apresentaram valores de hemoglobina $<11,0$ $\mathrm{g} / \mathrm{dL}$ com idade até 60 meses e $<11,5 \mathrm{~g} / \mathrm{dL}$ para crianças com idade superior a 60 meses.

As análises estatísticas utilizadas para avaliar o comportamento do percentual da anemia, bem como fatores (sexo e idade) que influenciam a ocorrência da doença nos anos 2001, 2005 e 2011 foram o teste qui-quadrado, intervalo de confiança, além do Odds ratio (OR). O software utilizado foi o $R$ versão 2.15.3 9 (R DEVELOPMENT CORE TEAM, 2012).

A Tabela 1 abaixo apresenta as frequencias de sexo e idade das crianças avaliadas no estudo para os anos 2001, 2005 e 2011. Pôde-se observar que a distribuição do sexo entre as crianças foi aparentemente similar entre os anos e apresentou percentuais próximos a $49 \%$ no sexo feminino, enquanto que para o sexo masculino esses percentuais eram de $51, \%$.

A distribuição da faixa etária das crianças demonstrou que no ano de $2001,17 \%$ das crianças tinham até 24 meses, 34\% tinham de 24 a 48 meses, $16 \%$ tinham 48 a 60 meses e $32 \%$ tinham mais que 60 meses. Para o ano de 2005 , aproximdamente, $10 \%$ das crianças tinham até 24 meses, $43 \%$ tinham de 24 a 48 meses e $23 \%$ tinham 48 a 60 meses e mais que 60 meses; e para 0 ano de $2011,4 \%$ das crianças tinham até 24 meses, $28 \%$ tinham de 24 a 48 meses e 48 a 60 meses e $39 \%$ tinham mais que 60 meses, aproximadamente.

Em relação à anemia, observou-se que a porcentagem de crianças anêmicas não apresentou variação considerável entre os anos, apresentando percentuais de $28,61 \%$ em 2001, $26,78 \%$ em 2005 e $23,64 \%$ para o ano de 2011. Dessa forma, observa-se que a tendência temporal da anemia apresentou uma pequena queda, não significativa, nos 11 anos avaliados. 
TABELA 1 Composição da amostra das crianças das creches do Distrito

Sanitário Leste de Belo Horizonte- MG nos anos 2001, 2005 e 2011.

\begin{tabular}{|c|c|c|c|c|c|c|c|c|c|}
\hline & \multirow{2}{*}{$\begin{array}{c}\text { Variáveis } \\
\text { Feminino }\end{array}$} & \multicolumn{2}{|r|}{2001} & \multicolumn{2}{|r|}{2005} & \multicolumn{2}{|r|}{2011} & \multicolumn{2}{|c|}{ Total } \\
\hline \multirow{3}{*}{ Sexo } & & 175 & $49,58 \%$ & 196 & $48,16 \%$ & 81 & $49,09 \%$ & 452 & $48,86 \%$ \\
\hline & Masculino & 178 & $50,42 \%$ & 211 & $51,84 \%$ & 84 & $50,91 \%$ & 473 & $51,14 \%$ \\
\hline & Total & 353 & $100,00 \%$ & 407 & $100,00 \%$ & 165 & $100,00 \%$ & 925 & $100,00 \%$ \\
\hline \multirow{6}{*}{ Idade } & Até 24 meses & 60 & $17,00 \%$ & 43 & $10,57 \%$ & 6 & $3,77 \%$ & 109 & $11,86 \%$ \\
\hline & 24 a 48 meses & 121 & $34,28 \%$ & 176 & $43,24 \%$ & 45 & $28,30 \%$ & 342 & $37,21 \%$ \\
\hline & 48 a 60 meses & 58 & $16,43 \%$ & 94 & $23,10 \%$ & 46 & $28,93 \%$ & 198 & $21,55 \%$ \\
\hline & Maior que 60 & & & & & & & & \\
\hline & meses & 114 & $32,29 \%$ & 94 & $23,10 \%$ & 68 & $38,99 \%$ & 270 & $29,38 \%$ \\
\hline & Total & 353 & $100,00 \%$ & 407 & $100,00 \%$ & 165 & $100,00 \%$ & 919 & $100,00 \%$ \\
\hline \multirow{3}{*}{ Anemia } & Não Anêmico & 252 & $71,39 \%$ & 298 & $73,22 \%$ & 126 & $76,36 \%$ & 676 & $73,08 \%$ \\
\hline & Anêmico & 101 & $28,61 \%$ & 109 & $26,78 \%$ & 39 & $23,64 \%$ & 249 & $26,92 \%$ \\
\hline & Total & 353 & $100,00 \%$ & 407 & $100,00 \%$ & 165 & $100,00 \%$ & 925 & $100,00 \%$ \\
\hline
\end{tabular}

A concentração sérica de hemoglobia das crianças do estudo foi avaliada entre os anos, podendo-se observar que não houve uma variação significativa desses valores (Tabela 2 e Gráfico 1).

TABELA 2-Medidas descritivas da concentração de hemoglobina das crianças prertencentes ás creches co Distrito Sanitário Leste de Belo Horizonte-MG, nos anos 2001, 2005 e 2011.

\begin{tabular}{lccccccccc}
\hline Anos & $\mathbf{N}$ & Média & D.P. & Mín. & $\mathbf{1}^{\mathbf{0}}$ & $\mathbf{2}^{\mathbf{0}}$ & $\mathbf{3}^{\circ} \mathbf{Q}$ & Máx. & Valor $\mathbf{P}^{\mathrm{a}}$ \\
\hline $\mathbf{2 0 0 1}$ & 353 & 11,77 & 1,39 & 6,40 & 10,90 & 11,90 & 12,70 & 17,70 & \\
$\mathbf{2 0 0 5}$ & 407 & 11,82 & 1,35 & 6,90 & 10,90 & 11,90 & 12,70 & 17,70 & 0,6623 \\
$\mathbf{2 0 1 1}$ & 165 & 11,95 & 1,38 & 8,60 & 11,00 & 11,90 & 12,80 & 16,60 & \\
\hline Geral & 925 & 11,81 & 1,38 & 6,40 & 10,90 & 11,90 & 12,70 & 17,70 & \\
\hline
\end{tabular}


Gráfico 1- Frequencia e boxplot da concentração de hemoglobina das crianças pré-escolares das creches do Distrito Leste de Belo Horizonte-MG, 2001, 2005e 2011
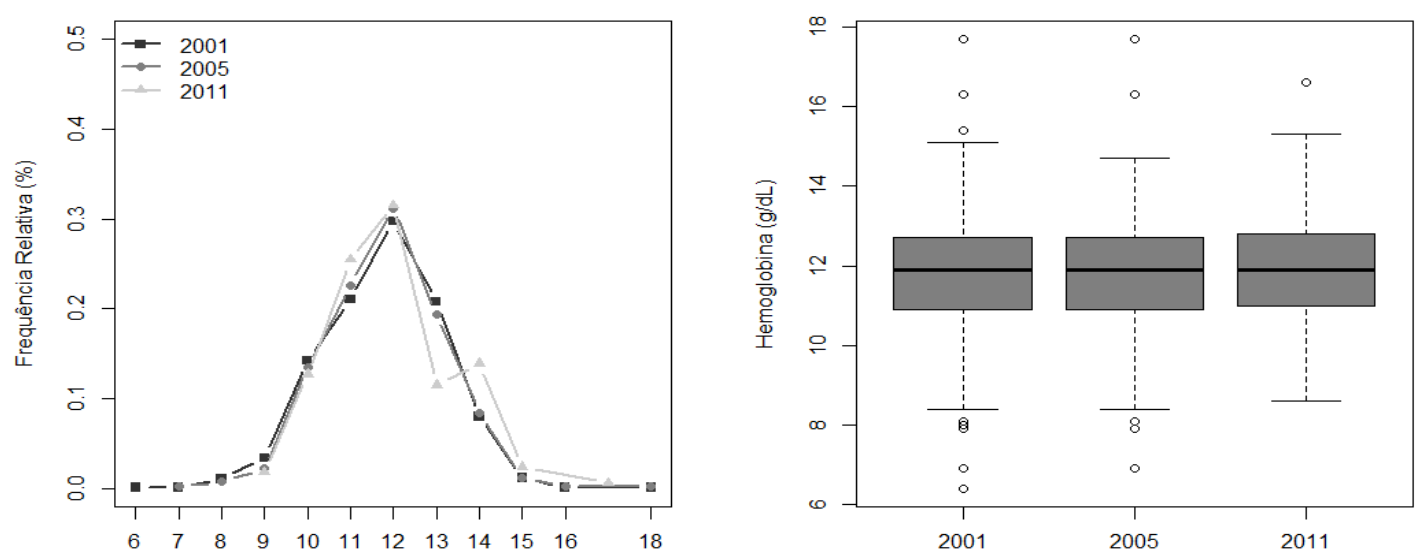

Fonte: Dados da pequisa

Comportamento da anemia foi avaliado em relação ao sexo e faixa etária das crianças, conforme apresentado nas Tabelas 3 e 4 e ilustrado no Gráfico 2 abaixo.

TABELA 3-Tendência temporal da anemia estratificada pelo sexo das crianças pré-escolares das creches do Distrito Leste de Belo Horizonte-MG, 2001, 2005 e 2011.

\begin{tabular}{|c|c|c|c|c|c|c|c|c|c|}
\hline \multirow[t]{2}{*}{ Sexo } & \multirow{2}{*}{$\begin{array}{l}\text { Anemia } \\
\text { Ausente }\end{array}$} & \multicolumn{2}{|r|}{2001} & \multicolumn{2}{|r|}{2005} & \multicolumn{2}{|r|}{2011} & Total & \multirow[t]{2}{*}{$\begin{array}{c}\text { Valor- } \\
\mathbf{P}^{\mathrm{a}}\end{array}$} \\
\hline & & 130 & $74,3 \%$ & 141 & $71,9 \%$ & 58 & $71,6 \%$ & $32972,8 \%$ & \\
\hline \multirow[t]{3}{*}{ Feminino } & Presente & 45 & $25,7 \%$ & 55 & $28,1 \%$ & 23 & $28,4 \%$ & $123 \quad 27,2 \%$ & 0,841 \\
\hline & Total & 175 & $100,0 \%$ & 196 & $100,0 \%$ & 81 & $100,0 \%$ & $452100,0 \%$ & \\
\hline & Ausente & 122 & $68,5 \%$ & 157 & $74,4 \%$ & 68 & $81,0 \%$ & $347 \quad 73,4 \%$ & \\
\hline \multirow[t]{2}{*}{ Masculino } & Presente & 56 & $31,5 \%$ & 54 & $25,6 \%$ & 16 & $19,0 \%$ & $126 \quad 26,6 \%$ & 0,095 \\
\hline & Total & 178 & $100,0 \%$ & 211 & $100,0 \%$ & 84 & $100,0 \%$ & $473100,0 \%$ & \\
\hline
\end{tabular}

\footnotetext{
${ }^{a}$ Teste qui-quadrado
} 
Observou-se que independentemente do sexo não houve evidência significativa de que o percentual de anemia tenha se modificado ao longo dos anos.

TABELA 4 -Tendência temporal da anemia estratificada pela faixa etária das crianças pré-escolares do Distrito Sanitário Leste de Belo HorizonteMG, 2001, 2005 e 2011.

\begin{tabular}{|c|c|c|c|c|c|c|c|c|c|c|}
\hline Idade & Anemia & & 2001 & & 2005 & & 2011 & & Total & $\begin{array}{c}\text { Valor- } \\
\mathbf{P}^{\mathrm{a}}\end{array}$ \\
\hline \multirow{3}{*}{$\begin{array}{l}\text { Até } 24 \\
\text { meses }\end{array}$} & Ausente & 25 & $41,7 \%$ & 12 & $27,9 \%$ & 3 & $50,0 \%$ & 40 & $36,7 \%$ & \multirow{3}{*}{$0,276^{b}$} \\
\hline & Presente & 35 & $58,3 \%$ & 31 & $72,1 \%$ & 3 & $50,0 \%$ & 69 & $63,3 \%$ & \\
\hline & Total & 60 & $100,0 \%$ & 43 & $100,0 \%$ & 6 & $100,0 \%$ & 109 & $100,0 \%$ & \\
\hline \multirow{3}{*}{$\begin{array}{l}24 \text { a } 48 \\
\text { meses }\end{array}$} & Ausente & 82 & $67,8 \%$ & 130 & $73,9 \%$ & 33 & $73,3 \%$ & 245 & $71,6 \%$ & \multirow{3}{*}{0,516} \\
\hline & Presente & 39 & $32,2 \%$ & 46 & $26,1 \%$ & 12 & $26,7 \%$ & 97 & $28,4 \%$ & \\
\hline & Total & 121 & $100,0 \%$ & 176 & $100,0 \%$ & 45 & $100,0 \%$ & 342 & $100,0 \%$ & \\
\hline \multirow{3}{*}{$\begin{array}{l}48 \text { a } 60 \\
\text { meses }\end{array}$} & Ausente & 44 & $75,9 \%$ & 74 & $78,7 \%$ & 36 & $78,3 \%$ & 154 & $77,8 \%$ & \multirow{3}{*}{0,904} \\
\hline & Presente & 14 & $24,1 \%$ & 20 & $21,3 \%$ & 10 & $21,7 \%$ & 44 & $22,2 \%$ & \\
\hline & Total & 58 & $100,0 \%$ & 94 & $100,0 \%$ & 46 & $100,0 \%$ & 198 & $100,0 \%$ & \\
\hline Maior & Ausente & 101 & $88,6 \%$ & 82 & $87,2 \%$ & 50 & $80,6 \%$ & 233 & $86,3 \%$ & \multirow{3}{*}{0,334} \\
\hline que 60 & Presente & 13 & $11,4 \%$ & 12 & $12,8 \%$ & 12 & $19,4 \%$ & 37 & $13,7 \%$ & \\
\hline meses & Total & 114 & $100,0 \%$ & 94 & $100,0 \%$ & 68 & $100,0 \%$ & 270 & $100,0 \%$ & \\
\hline
\end{tabular}

${ }^{a}$ Teste qui quadrado; b Teste exato de Fisher

Ao avaliar a ocorrência de anemia estratificada pela faixa etária das crianças observa-se que não houve diferença significativa entre as faixas etárias nos anos avaliados. Ou seja, independente da faixa etária da criança não existe evidência de modificação do percentual de anemia ao longo dos anos.

Ressalta-se, ainda, que a anemia apresentou-se mais prevalente em crianças pertencentes à faixa etária até 24 meses, tendo sido demonstrado um decréscimo da prevalência da mesma com o aumento da faixa da criança. 
Os Gráfico 2 abaixo ilustra a tendência da anemia de acordo com o sexo e faixa etária das crianças, entre os anos 2001, 2005 e 2011. Pôde-se observar que de acordo com estas variáveis o percentual de anemia nos 11 anos estudados tendeu para a linearidade, não havendo diferença significativa entre os anos.

\section{Gráfico 2- Percentual da anemia das crianças pré-escolares do Distrito} Sanitário Leste de Belo Horizonte-MG, estratificado pelo sexo e faixa etária nos anos 2001, 2005 e 2011.
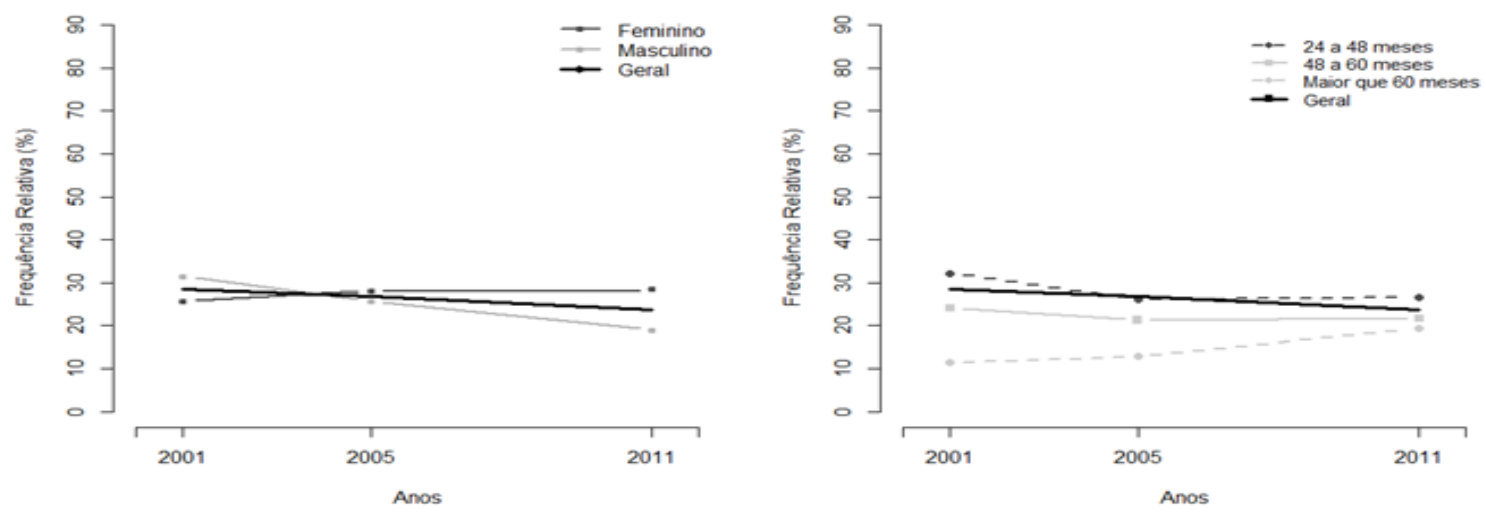

Fonte: Dados da pesquisa

Os resultados encontrados no presente trabalho demonstram a tendência temporal de estabilidade nos percentuais de anemia nas crianças de creches públicas do município de Belo Horizonte - MG. Observa-se que não houve uma redução significativa nos 11 anos avaliados, sendo de 28,61\% em 2001, 26,78\% em 2005 e 23,64\% em 2011, considerando a doença um importante problema de saúde pública. A Organização Mundial da Saúde (OMS) classifica a significância populacional da anemia como aceitável abaixo de $5 \%$, leve de 5 a 19,9\%, moderada de 20 a 39,9\% e grave maior ou igual a $40 \%$. A prevalência da anemia em países industrializados é considerada ainda inaceitável atingindo valores de 5 a $16 \%$ enquanto que em países em desenvolvimento este número varia de 30 a $45 \%$, valores estes próximos aos encontrados no presente estudo traduzindo a gravidade da doença nesses locais (FILHO; BRESANI, 2008). 
Observou-se que a média de hemoglobina não sofreu variação significativa nos anos avaliados. Estudo realizado com crianças pertencentes a quatro municípios diferentes do Nordeste apresentou um percentual de $73 \%$ de anemia e média de hemoglobina de $9,8 \mathrm{~g} / \mathrm{dL}$, demonstrando a elevada prevalência e reduzida média de hemoglobina em lactentes (LIMA, et al., 2004). Outro estudo também realizado com crianças pré-escolares observou um valor de hemoglobina reduzido (média 9,5 g/dL) ao avaliar a adesão das mesmas à suplementação com sulfato ferroso (EICKMANN, et al., 2008).

No Brasil, existem relatos na literatura que demonstraram a intensificação dos esforços para controle da anemia por parte dos órgãos governamentais desde 1990, quando estudos foram desenvolvidos com o intuito de reduzir a ocorrência dão distúrbio nutricional em gestantes e crianças em idade pré-escolar (JORDÃO et al., 2009; SZARFARC, 2010). Entretanto, os referidos relatos não apresentavam medidas indicativas de intervenções efetivas para o controle da anemia.

As crianças, em especial na primeira infância, apresentam-se como faixa de risco para o acometimento da anemia, uma vez que estão no período de desmame, modificam a alimentação pela introdução da alimentação complementar, ingressam no ambiente escolar com modificação e introdução de novos hábitos alimentares, estão sujeitos a quadros de infecções e conseqüente diarréia, o que compromete a absorção do mineral ferro e aumenta a chance de ocorrência da anemia (CUERVO, et al., 2005).

No presente estudo observou-se uma maior prevalência da anemia na faixa etária até 24 meses. Além disso, observou-se que a prevalência da anemia decresce com o aumento da idade. Tal fato já era esperado, uma vez que os primeiros anos de vida representam forte fator de risco para o aumento da prevalência da anemia, em virtude das modificações alimentares da criança. Neste período ocorre a transição entre o aleitamento materno e a introdução da alimentação complementar o que pode contribuir para o aparecimento da anemia, uma vez que os estoques de ferro no lactente já foram exauridos e a introdução de novos alimentos pode não suprir as necessidades da criança, em relação aos micronutrientes, especialmente o ferro. Além disso, os dois 
primeiros anos de vida constituem período de extrema vulnerabilidade da criança já que esta se encontra em período de rápido crescimento e necessita de aporte nutricional e energético adequado para o desenvolvimento do sistema imunitário contra infecções (GARCIA, et al., 2011).

A importância do combate à anemia em crianças já foi demonstrada em diversos trabalhos, tendo em vista suas consequências para a criança como maior susceptibilidade às infecções, maior mortalidade, atraso no crescimento e desenvolvimento e maior permeabilidade intestinal com tendência a maior sensibilização a alérgenos. Além disso, outros estudos demonstraram que a anemia na infância se relaciona também com baixos escores em testes de desenvolvimento mental, de linguagem e atividade motora, podendo levar a seqüelas irreversíveis, mesmo com tratamento adequado (UMBELINO; ROSSI, 2006).

Para o Brasil a prevalência da anemia aumenta as despesas públicas com a saúde direta e indiretamente, já que dados de 2008 estimam que cerca de $R \$ 116$ milhões foram gastos para tratar agravo pela deficiência de ferro, além das conseqüências da anemia para as crianças conforme já mencionado (EDNA, et al., 2010). Diante da relevância do combate à anemia, a avaliação das estratégias governamentais de combate à doença é importante tanto para o estudo dos pontos falhos destas estratégias como para o aprimoramento de novas medidas de controle da prevalência da anemia.

Embora já tenham sido desenvolvidos programas com o intuito de redução da prevalência da anemia existe uma deficiência em dados de tendência temporal da mesma, em especial no Estado de Minas Gerais. Tais estudos contribuem para a associação entre os percentuais de anemia em diferentes momentos com a avaliação da efetividade das estratégias de controle da mesma. Observa-se uma escassez destes estudos já que a prevalência da anemia ainda permanece elevada. Em 2002, o Ministério da Saúde deliberou a fortificação das farinhas consumidas no país com micronutrientes. Foi determinada a obrigatoriedade da fortificação das farinhas de trigo e milho com ferro e ácido fólico definido na Resolução RDC 344/02 (ANVISA, 2002). De acordo com esta resolução cada $100 \mathrm{~g}$ de farinha deve 
fornecer 4,2 mg de ferro e $150 \mathrm{mcg}$ de ácido fólico. O ano de 2001 representa um marco no período anterior à fortificação das farinhas de trigo e milho, quando comparado com o ano de 2005 em que as farinhas consumidas pela população já eram fortificadas com o ferro e ácido fólico. Entretanto, o percentual de anêmicos em 2001 foi próximo (28,61\%) ao encontrado em 2005 $(26,78 \%)$ e ainda, apresentou uma redução pouco considerável nove anos após a adoção da fortificação das farinhas (23,64\%).

Apesar de que algumas das experiências nacionais e internacionais a respeito da fortificação de farinhas com ferro no combate à anemia em crianças tenham sido positivas (COSTA et al., 2009; TUMA et al., 2003), no Brasil, um estudo realizado com crianças na faixa etária de zero a 71 meses, um e dois anos após o consumo de farinha fortificada não encontrou redução nos níveis de hemoglobina das crianças (ASSUNÇÃO et al., 2009). Diversas razões podem estar envolvidas com este resultado como, por exemplo, o baixo consumo de alimentos que contenham a farinha fortificada, a biodisponibilidade do ferro adicionado à farinha, a adequação da quantidade de ferro adicionada, bem como o hábito alimentar da criança, em virtude da possibilidade de consumo de alimentos que prejudiquem a absorção do mineral ferro (TUMA, et al., 2003).

Outra estratégia adotada pelo governo brasileiro foi o Programa Nacional de Suplementação de Ferro (PNSF) em crianças de 6 a 18 meses (ASSUNÇÃO et al., 2009). Resultados positivos obtidos no município de São Paulo com a administração de dose única semanal do sulfato ferroso para as crianças foram importantes para a adoção de tal iniciativa em todo o país, estrategicamente no projeto para controle da anemia ferropriva em menores de dois anos. Embora alguns resultados tenham sido relevantes, como o controle no nível de hemoglobina, relato pelas mães do efeito benéfico da suplementação semanal do ferro, adesão plena e parcial razoavelmente significativa, existem ainda diversos pontos negativos para a efetividade do programa. Assim, a baixa biodisponibilidade do ferro empregado na fortificação, o sabor metálico do mineral, bem como efeitos colaterais da sua utilização como náuses, diarréia ou constipação e dores abdominais constituem fatores 
que justificam a rejeição a suplementação do ferro e efetividade do referido programa (SZARFARC, 2010).

O presente estudo representa um incentivo aos órgãos públicos para a criação de estratégias que avaliem a eficácia dos programas de controle da anemia, já que em um período de 11 anos a prevalência da doença, praticamente não sofreu alteração passando de $27 \%$ para $23 \%$, aproximadamente. Existem diversos fatores que podem influir no resultado dos programas adotados para combate da anemia em crianças como, por exemplo, o hábito alimentar, condições higiênicas sanitárias, escolaridade dos pais, consumo de alimentos fortificados dentre outros.

A fortificação de alimentos é uma estratégia já adotada em inúmeros países e que merece destaque. Uma das principais razões da facilidade de adoção de alimentos fortificados é a manutenção do hábito alimentar da população e a redução dos efeitos colaterais e toxicidade já que as doses do mineral utilizado são mínimas (ROCHA, et al., 2011). Diversos alimentos já foram avaliados quanto à fortificação com o ferro como o leite em pó, biscoito, açúcar, cereal à base de arroz, pão preto, água, dentre outros. Um fator importante que deve ser levado em consideração na escolha do alimento é o consumo habitual pela população bem como a biodisponibilidade do ferro na forma em que é oferecido e consumido. Além disso, surge a necessidade da avaliação da eficácia da intervenção bem como validade dos estudos que comprovem tal eficácia (LAMOUNIER et al., 2010).

A fortificação de alimentos comumente consumidos pelas crianças é uma alternativa interessante para a prevalência da anemia. A busca por alimentos suplementados que possam contemplar as necessidades de ferro das crianças, tendo em vista o consumo frequente destes alimentos, constitui estratégia relevante no contexto da anemia. Assim, trabalho realizado em Belo Horizonte - MG com a fortificação da água com ferro e vitamina $C$ obteve resultados importantes na redução da prevalência da anemia em crianças de idade de 6 a 74 meses. Neste estudo, foi apresentada uma redução de 29,3\% para $7,9 \%$ de anemia nas crianças pertencentes a creches públicas, assistidas 
pelo programa de suplementação do mineral ferro na água (ROCHA et al., 2011).

A anemia ferropriva diferentemente de outros distúrbios nutricionais como, por exemplo, a desnutrição que apresenta acentuada queda nas últimas décadas, permanece elevada em diversos segmentos da população com destaque para as crianças e gestantes. Assim, considera-se importante a avaliação da efetividade de programas públicos no controle da anemia como avaliação da adesão da população ao tratamento medicamentoso, o desenvolvimento de produtos fortificados relevantes e consumidos habitualmente pela população, além de políticas que permitam o acesso ao alimento igualitário à população com o intuito de não apenas reduzir a prevalência, mas erradicação da anemia.

Uma incógnita ainda permanece constante, já que trabalhos vêm demonstrando que fatores anteriormente relacionados com a ocorrência de distúrbios nutricionais na infância, como por exemplo, a renda familiar, não tem apresentado relação direta com a ocorrência destes distúrbios. Ademais, o Brasil apresenta, atualmente, uma nítida melhora das condições socioeconômicas da população e acesso aos serviços de saúde o que contribuiu no ano de 2013 para a redução considerável da taxa de mortalidade infantil (ASSUNÇÃO et al., 2009). Entretanto, a anemia ainda mantém a prevalência constante no país, assim, quais estratégias poderiam ser adotadas ou ainda, se seria possível uma relação fisiológica da anemia entre as crianças, não sabemos ainda, qual direção adotar como medida assertiva de controle da doença. Os dados da tendência da anemia vêm confirmar os achados de linearidade da prevalência da doença, entretanto, estudos que avaliem a efetividade dos programas e propostas de controle da anemia se fazem necessários.

\section{Conclusão}

A tendência temporal da anemia em crianças pré-escolares de Belo Horizonte - MG foi de estabilidade da doença em um período de 11 anos. Além 
disso, não houve associação significativa entre os gêneros ao longo dos anos. A faixa etária da criança também não sofreu modificação ao longo dos anos em relação ao percentual de anemia. Nos três cortes avaliados, observou-se um decréscimo da anemia com o aumento da faixa etária. Os resultados do presente estudo demonstram a necessidade de avaliação dos programas já desenvolvidos de combate à anemia ferropriva em crianças bem como o incentivo ao desenvolvimento de novas estratégias para o controle da doença.

\section{Referências}

Agência Nacional de Vigilância Sanitária. RDC n.344, de 13 dez. 2002. Disponível

em: http://www.anvisa.gov.br/legis/resol/2002/344_02rdc.htm>Acesso em 05 mai. 2011.

ASSUNÇÃO, M. C. F; SANTOS I.S, BARROS A. J. D, GIGANTE D. P. VICTORA, C. G. Efeito da fortificação de farinhas com ferro sobre anemia em pré-escolares, Pelotas, RS, Soc. Food Nutr, v.34, n.1, p.59-74, 2009.

BRASIL, Ministério da Saúde. Secretaria de Atenção à Saúde. Departamento de Atenção Básica. Manual operacional do Programa Nacional de Suplementação de Ferro. Brasília; 2005 (Série A. Normas e Manuais Técnicos). Disponível em:

http://dtr2004.saude.gov.br/nutricao/documentos/manual ferro.pdf.>Acesso em mai 2011.

COSTA, C. A.; MACHADO, E. H.; COLLI, C.; LATORRE, W. C.; SZARFARC, S. C. Anemia em pré-escolares atendidos em creches de São Paulo (SP): perspectivas decorrentes da fortificação das farinhas de trigo e de milho. Nutrire, Brasília, v.34, p. 59-74, 2009. 
CUERVO, M.R; AERTES, D. R. G. C.; HALPERN, R. Vigilância do estado nutricional das crianças de um distrito de saúde no Sul do Brasil. Jornal de Pediatria, Rio de Janeiro, v.81, p. 325-331, 2005.

EICKMANN, S. H. Efetividade da suplementação semanal com ferro sobre a concentração de hemoglobina, estado nutricional e o desenvolvimento de lactentes em creches do Recife, Pernambuco, Brasil. Cad. Saúde Pública, Rio de Janeiro, v.24, n.2, p.303-311, 2008.

ENSGSTROM, E. M. Efetividade da suplementação diária ou semanal com ferro na prevenção da anemia em lactentes. Rev Saúde Pública, v.42, p. 786795, 2008.

FILHO, M. B.; SOUZA, A. I.; BRESANI, C. C. Anemia como problema de saúde pública: uma realidade atual. Ciência \& Saúde Coletiva, v.13, n.6, p.19171922, 2008.

GARCIA, M. T.; GRANADO, F. S.; CARDOSO, M. A. Alimentação complementar e estado nutricional de crianças menores de dois anos atendidas no Programa Saúde da Família em Acrelândia, Acre, Amazônia Ocidental Brasileira. Cad. Saúde Pública, Rio de Janeiro, v.27, n.2, p.305-316, 2011.

GISELA S. B.; GUIMARÃES, L. V.; FISBERG, M. Anemia em crianças menores de 3 anos que freqüentam creches públicas em período integral. Jornal de Pediatria, Rio de Janeiro, v.78, n.1, p. 50-56.

JORDÃO, R. E.; BERNARDI, J. I. D.; AZEVEDO, B. F. A. Prevalência de anemia ferropriva no Brasil: uma revisão sistemática. Revista Paulista de Pediatria, v.27, n.1, p. 90-98, 2009.

LAMOUNIER, J. A.; CAPANEMA, F. D. ROCHA, D. S. OLIVEIRA, J. E. D. SILVA, M.C. Iron Fortification Strategies for the Control of Childhood Anemia in Brazil. Journal of Tropical Pediatrics v, 56, p. 448-451, 2010.

LIMA, A. C. V. M. de S; CABRAL, L. P. I.; MELLO, R. S. A.; HELENA, E. S.; DILMA, P. M.; CARVALHO, L. M. Fatores determinantes dos níveis de hemoglobina em crianças aos 12 meses de vida na Zona da Mata Meridional 
de Pernambuco. Revista Brasileira de Saúde Materna Infantil, v.4, n.1, p.3543, 2004.

LOPEZ, A. D.; MATHERS, C. D. EZZATI, M.; JAMISON, D. T.; MURRAY, C. J. L. Global and regional burden of disease and risk factors: systematic analysis of population health data. The Lancet, v, 367, mai., 2006.

MACHADO, E. H. S.; BRASIL, A. L. D.; PALMA, D. Condição nutricional e prevalência de anemia em crianças matriculadas em creches beneficentes. Revista Paulista de Pediatria, v.23, n.1, p. 21-26, 2005.

OMS. Worldwide prevalence of anemia 1993-2005. Genebra: WHO, 2008.

POLLIT, E. Early iron deficiency and later mental retardation. The American Journal of Clinical Nutrition, v.69, p. 4-5, 1999.

TEAM, R. D. C. A Language and Environment for Statistical Computing. Viena, Austria: R Foundation for Statistical Computing. http://www.R-project.org/.2012., 2012.

ROCHA, D. S.; CAPANEMA, F. D.; NETTO, M. P.; AlMEIDA, C. A. N.; FRANCESCHINI, S. C. C.; LAMOUNIER, J. A. Effectiveness of fortification of drinking water with iron and vitamin $C$ in the reduction of anemia and improvement of nutritional status in children cared for in day care centers of the city of Belo Horizonte, Brazil. Food and Nutrition Bulletin (Tokyo. Print), v.32, p. 340-346, 2011.

SILVA D. G. Risk factors for anemia in infants assisted by public health services: the importance of feeding practices and iron supplementation. Jornal of Pediatric, Rio de Janeiro, v.83, n.2, p. 149-156.

SILVA, E. H.; SZARFARC, S.C.; CYRILLO, D.C.; FUJIMORI, E.; COLLI, C. Reflexão sobre as estratégias de controle da anemia em gestantes no Brasil. Segurança Alimentar e Nutricional, Campinas, v.17, n.1, p.104-112, 2010. 
SPINELLI, M. G. N.; MARCHIONI, D. M. L.; SOUZA, J. M. P.; SOUZA, S. B.; SZARFARC, S. C. Fatores de risco para anemia em crianças de 6 a 12 meses no Brasil. Revista Panam Salud Publica, v, 17, n.2, p. 84-91, 2005.

SZARFARC, S. C. Políticas públicas para o controle da anemia ferropriva. Revista Brasileria de Hematologia, v. 32, p. 2-7, 2010.

TUMA, Rahilda Brito et al. Impacto da farinha de mandioca fortificada com ferro aminoácido quelato no nível de hemoglobina de pré-escolares. Revista de Nutrição, v.16, p. 29-39, 2003.

UMBELINO, D.C.; ROSSI, E. A. Deficiência de ferro: conseqüências biológicas e propostas de prevenção. Revista Ciência Farmarmacêutica Básica, v. 27, n.2, p.103-112, 2006.

WHO, World Health Organization Feeding and Nutrition of Infants and Young Children - Regional Publications, n 87. Copenhagen: World Health Organization, 2003. 